
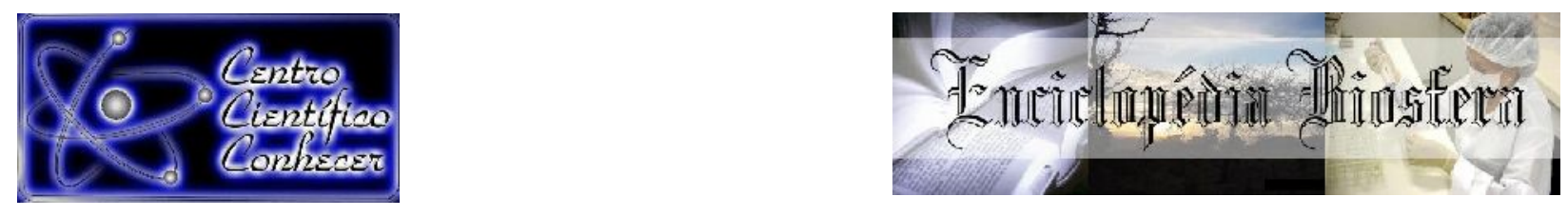

\title{
MASSA ESPECÍFICA BÁSICA, APARENTE SATURADA E TEOR DE UMIDADE DA MADEIRA DE Myrcianthes pungens (O. Berg) D. Legrand
}

\author{
Luana Candaten ${ }^{1}$; Eduarda Bandera ${ }^{2}$ Tauana de Souza Mangini ${ }^{2}$ Laura da Silva \\ Zanchetta $^{2}$ Rômulo Trevisan ${ }^{3}$ \\ ${ }^{1}$ Mestranda em Recursos Florestais pela Universidade de São Paulo - Escola \\ Superior de Agricultura Luiz de Queiroz, e-mail: Icandaten@usp.br. \\ ${ }^{2}$ Graduandas em Engenharia Florestal pela Universidade Federal de Santa Maria - \\ Campus Frederico Westphalen, \\ ${ }^{2}$ Professor Doutor da Universidade Federal de Santa Maria - Campus Frederico \\ Westphalen.
}

Recebido em: 06/04/2019 - Aprovado em: 10/06/2019 - Publicado em: 30/06/2019 DOI: 10.18677/EnciBio_2019A85

\begin{abstract}
RESUMO
O estudo das propriedades físicas da madeira torna-se essencial para a correta aplicação desta em vários âmbitos como, por exemplo, na construção civil ou na indústria moveleira. O objetivo deste artigo foi analisar a massa específica básica, aparente saturada e o teor de umidade da madeira de Myrcianthes pungens (O.Berg) D. Legrand, bem como a variação radial e longitudinal no fuste. Para realização do trabalho utilizou-se uma árvore da espécie, após o abate, foram confeccionados discos nas posições da base $(0,10 \mathrm{~m})$, diâmetro a $1,30 \mathrm{~m}$ do solo (DAP), 25, 50, 75 e 100\% em função da altura comercial, afim de verificar o comportamento das propriedades físicas da mesma. Destes foram seccionadas cunhas, contendo a medula, bem como partes do cerne e do alburno. Para o sentido medula-casca os dados foram obtidos a partir de filetes de $1 \times 1 \mathrm{~cm}$, identificados, marcados e retirados do material restante. A determinação das massas específicas em ambos os sentidos, assim como a determinação do teor de umidade, seguiu a norma ASTM D143-94 (1995). A partir das aferições realizadas foi possível verificar que o valor ponderado de massa específica básica foi de $0,725 \mathrm{~g} / \mathrm{cm}^{3}$, classificando a madeira de Guabiju como média. Já a ponderação da massa específica aparente saturada ao teor de umidade de $72,7 \%$ foi de $1,254 \mathrm{~g} / \mathrm{cm}^{3}$. Com exceção do teor de umidade, os resultados indicaram que as propriedades físicas estudadas foram influenciadas pelas posições no tronco, onde ambas as massas específicas tiveram uma redução da base para o topo e da medula para a casca.
\end{abstract}

PALAVRAS-CHAVE: propriedades físicas; espécie nativa; variação radial e longitudinal. 


\title{
BASIC SPECIFIC GRAVITY, APPARENT AND MOISTURE CONTENT OF THE WOOD OF Myrcianthes pungens (O. Berg) D. Legrand
}

\begin{abstract}
The study of the physical properties of wood is essential for the correct application of this in various fields, for example, in the civil construction or furniture industry. The objective of this article was to analyze the basic specific gravity, apparent and moisture content of the wood of Myrcianthes pungens (O. Berg) D. Legrand, as well as the radial and longitudinal variation in the stem. A tree of the species, after the harvested, discs from base, in the diameter at $1.30 \mathrm{~m}$ height of soil $(\mathrm{DBH}), 25,50$, 75 , and $100 \%$ depending on the commercial height, in order to verify the behaviour of its physical properties. From these they were sectioned in wedges containing pith, heartwood, and sapwood. From the vascular cambium to the medulla the data were obtained from $1 \times 1 \mathrm{~cm}$ fillets, identified, marked and removed from the remaining material. Determination of the specific masses in both directions, as well as determination of the moisture content, followed the ASTM standard D143-94 (1995). From the realized measurements it was possible to verify that the basic density value weighted value was $0.725 \mathrm{~g} / \mathrm{cm}^{3}$, classifying the Guabiju wood as mean. The weighting of the apparent specific mass saturated to the moisture content of $72.7 \%$

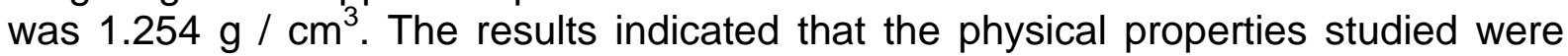
influenced by the positions in the trunk, where both specific masses had a reduction from the bottom-top and pith-bark.
\end{abstract}

KEYWORDS: physical properties; native species; radial and longitudinal variation.

\section{INTRODUÇÃo}

A madeira se destaca por possuir características orgânicas, heterogêneas e ser um elemento composto basicamente de celulose, hemiceluloses, lignina e extrativos (TRAUTENMÜLLER et al., 2014). A partir desses parâmetros, são necessárias pesquisas para obtenção de informações precisas que abordem espécies nativas e suas propriedades físicas e tecnológicas, facilitando a implantação das mesmas em diversos ramos do mercado.

Com isso, o estudo de espécies como a Myrcianthes pungens (O.Berg) D. Legrand, conhecida popularmente por Guabiju e pertencente à família Myrtaceae (WILSON et al., 2001), é indispensável em função da vasta presença dessa árvore na região Sul do Brasil. Tal conhecimento é de suma importância, pois inúmeras são as vantagens das espécies nativas quando comparadas às exóticas, principalmente tratando-se de propriedades físicas (MATTOS et al., 2011).

Dentre as caracterizações tecnológicas passíveis de aplicação em laboratório, Bonduelle et al. (2015) destacaram que, a massa específica é considerada um parâmetro para classificação do material, sendo a mais importante das propriedades físicas. Assim, essa faz uma relação do peso e volume do objeto estudado, associando a quantidade de espaços vazios existentes numa determinada porção do lenho (OSBORNE, 2016; DIAS, 2017; ZAQUE et al., 2018). Esse atributo influencia diretamente na resistência do produto e na transformação industrial (BONDUELLE et al., 2015) possibilitando a determinação da qualidade da madeira.

A massa específica pode variar entre e dentre espécies e ainda, no mesmo indivíduo, apresentando valores e comportamentos diferentes nas posições medulacasca e base-topo (ZAQUE et al., 2018). Os mesmos autores abordaram que a sua caracterização e, principalmente, a variação no fuste, tanto da medula para a casca, 
quanto da base para topo, associada à idade, são parâmetros essenciais para a designação do produto. Com isso, é fundamental, quando se estuda a massa específica das espécies, a avaliação radial e longitudinal no lenho, podendo a partir dos resultados, caracterizar a madeira e indicar o uso mais adequado.

Outro aspecto fundamental é o teor de umidade definido como a relação entre a massa da água contida na madeira e a massa seca do material, conforme a norma NBR 7190 (ABNT, 1997). Essa característica, juntamente com a massa específica, são propriedades físicas diretamente relacionadas, em que, a quantidade de espaços vazios é consequência da porcentagem de líquido presente no material. Sendo assim, esta parcela de água constituinte é um fator determinante na massa específica da espécie analisada.

A partir desses apontamentos e em consequência da escassa literatura relacionada às propriedades físicas de espécies nativas, como o caso do Guabiju, o presente trabalho teve como objetivo analisar a massa específica básica, aparente saturada e o teor de umidade da madeira de Myrcianthes pungens (O.Berg) D. Legrand, bem como a variação radial e longitudinal no fuste.

\section{MATERIAL E MÉTODOS}

Para a condução desse estudo foi utilizado um indivíduo adulto de Myrcianthes pungens o qual, após o abate, foi seccionado em discos retirados das posições da base $(0,10 \mathrm{~m}), 25,50,75,100 \%$ da altura comercial obtida a partir da presença do primeiro galho vivo e no diâmetro à altura de $1,30 \mathrm{~m}$ do solo (DAP). Posteriormente, os mesmos foram transportados até o Laboratório de Tecnologia de Produtos Florestais da Universidade Federal de Santa Maria - campus Frederico Westphalen, os quais passaram por uma identificação individual.

Após os procedimentos citados, o material foi imerso em água até que obtivesse a saturação total das fibras. Subsequentemente, dividiu-se o conteúdo em cunhas simetricamente opostas, contendo a medula e, também, partes do cerne e alburno, para a determinação das propriedades físicas no sentido longitudinal. Já, para o radial, as amostras foram confeccionadas a partir das cunhas restantes onde foram identificados, marcados e seccionados filetes de $1 \times 1 \mathrm{~cm}$.

Para a determinação da massa especifica básica (Equação 1), o peso a 0 \% de umidade foi obtido utilizando uma estufa com circulação de ar forçada a $103^{\circ} \mathrm{C}$ até a completa estabilização. Já o volume saturado das peças foi estabelecido através do método da balança hidrostática descrito por VITAL (1984), após completa saturação das amostras em água.

$$
\mathrm{Me}_{\mathrm{Básica}}=\frac{\mathrm{P}_{\mathrm{O} \%}}{\mathrm{~V}_{\mathrm{Y}}}
$$

Em que: $\mathrm{Me}_{\text {Básica }}=$ Massa específica básica $\left(\mathrm{g} / \mathrm{cm}^{3}\right) ; \mathrm{P}_{0 \%}=$ peso seco a $0 \%(\mathrm{~g}) ; \mathrm{V}_{\mathrm{V}}=$ Volume saturado $\left(\mathrm{cm}^{3}\right)$.

A obtenção dos valores de massa específica aparente saturada foi determinada por meio do peso saturado, ou seja, em condição de saturação total das fibras em água, e volume saturado, expressos pela Equação 2:

$$
\mathrm{Me}_{\text {Aparente sat }}=\frac{\text { Psat }}{\text { Vsat }}
$$

Em que: $\mathrm{Me}_{\text {Aparente sat }}=$ Massa específica aparente na condição saturada $\left(\mathrm{g} / \mathrm{cm}^{3}\right) ; P=P e s o$ na condição saturada $(\mathrm{g}) ; \mathrm{V}_{\mathrm{x}}=$ Volume na condição saturada $\left(\mathrm{cm}^{3}\right)$. 
A fim de calcular a massa específica básica e aparente saturada na condição ponderada, utilizaram-se as massas específicas ao longo do fuste, os volumes sem casca entre essas posições e o volume comercial sem casca da árvore, conforme Equação 3:

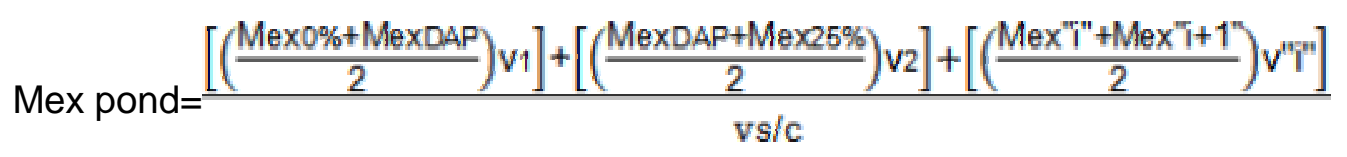

Em que: Mex pond = massa específica, básica ou aparente saturada, ponderada em função do volume comercial sem casca da árvore $\left(\mathrm{g} / \mathrm{cm}^{3}\right)$; Mex "i" = massa específica básica ou aparente saturada na posição "i" $\left(\mathrm{g} / \mathrm{cm}^{3}\right)$; v1, v2, v"i" = volume sem casca correspondente a duas posições sucessivas $\left(\mathrm{cm}^{3}\right) ; \mathrm{vs} / \mathrm{c}=$ volume sem casca da árvore $\left(\mathrm{cm}^{3}\right)$.

A importância do cálculo da massa específica ponderada, está na representatividade da mesma, que leva em consideração demais parâmetros do tronco.

Para obtenção do teor de umidade, utilizou-se a Equação 4:

$$
T u=\frac{P_{u-P_{0 \%}}}{P_{0 \%}} \times 100
$$

Onde: $\mathrm{Tu}=$ Teor de umidade saturado (\%); $\mathrm{P}_{\mathrm{u}}=$ Peso saturado $(\mathrm{g}) ; \mathrm{P}_{0 \%}=$ peso seco a $0 \%(g)$.

Os dados de massa específica básica e aparente saturada em cada posição relativa no sentido base-topo e medula-casca foram submetidos à análise de regressão utilizando o pacote estatístico SAS (Statistical Analysis System) e procedimento Stepwise para seleção de variáveis. O modelo foi definido pela Equação $5 \mathrm{com}$ base no coeficiente de determinação ajustado $\left(R^{2}{ }_{\text {aj. }}\right)$, erro padrão da estimativa $\left(\mathrm{S}_{\mathrm{xy}}\right)$, valor de $\mathrm{F}$ e análise da distribuição dos resíduos.

$$
\text { MEb;MEas; TU }=\mathrm{f}\left(\mathrm{P} ; \frac{1}{\mathrm{P}} ; \mathrm{P}^{2} ; \frac{1}{\mathrm{P}^{2}} ; \operatorname{In} \mathrm{P} ; \frac{1}{\ln \mathrm{P}} ; \sqrt{\mathrm{P})}\right.
$$

Onde: $\mathrm{MEb}=$ massa específica básica; MEas= massa específica aparente saturada, em $\mathrm{g} / \mathrm{cm}^{3} ; \mathrm{TU}=$ teor de umidade em $\% \mathrm{P}=$ posição relativa sentido base-topo em \%.

\section{RESULTADOS E DISCUSSÃO}

Os valores médios obtidos para teor de umidade, massa específica básica e aparente saturada ao longo do fuste da árvore de Myrcianthes pungens podem ser observados na Tabela 1. Os resultados permitiram evidenciar que o maior teor de umidade foi na posição $100 \%$ da altura comercial enquanto que, para as massas específicas básica e aparente saturada, ao contrário da umidade, os menores valores foram verificados nessa posição, além de não serem observadas amplitudes consideráveis. 
TABELA 1 - Resultados das médias de massa específica básica, massa específica aparente saturada e teor de umidade por posição no sentido axial de Myrcianthes pungens.

\begin{tabular}{cccc}
\hline $\begin{array}{c}\text { Posição } \\
\text { base-topo }\end{array}$ & $\begin{array}{c}\text { Massa específica } \\
\text { básica }\end{array}$ & $\begin{array}{c}\text { Massa específica } \\
\text { aparente saturada }\end{array}$ & Teor de umidade \\
\hline \multirow{2}{*}{$0,1 \mathrm{~m}$} & 0,732 & 1,330 & 58,1 \\
& $( \pm 0,078)$ & $( \pm 0,004)$ & $( \pm 14,8)$ \\
DAP & 0,774 & 1,231 & 64,3 \\
& $( \pm 0,045)$ & $( \pm 0,009)$ & $( \pm 1,1)$ \\
$25 \%$ & 0,735 & 1,268 & 72,6 \\
& $( \pm 0,012)$ & $( \pm 0,003)$ & $( \pm 3,3)$ \\
$50 \%$ & 0,760 & 1,255 & 66,3 \\
& $( \pm 0,007)$ & $( \pm 0,007)$ & $( \pm 1,17)$ \\
$75 \%$ & 0,702 & 1,243 & 77,2 \\
& $( \pm 0,006)$ & $( \pm 0,015)$ & $( \pm 0,68)$ \\
$100 \%$ & 0,559 & 1,215 & 97,8 \\
& $( \pm 0,019)$ & $( \pm 0,019)$ & $( \pm 16,9)$ \\
\hline
\end{tabular}

Onde: $\mathrm{DAP}=$ diâmetro à altura de $1,30 \mathrm{~m}$ do solo. Valores entre parênteses indicam o desvio padrão em $\mathrm{g} / \mathrm{cm}^{3}$ para as massas específicas e \% para teor de umidade.

Essa relação inversa entre o teor de umidade e a massa específica, verificada na Tabela 1, está em conformidade com a literatura e ocorre tanto em espécies exóticas quanto em nativas (FRIEDERICHS et al., 2015; JUIZO et al., 2015). Conforme estudo realizado por Candaten et al. (2018), avaliando as propriedades físicas da madeira de Maclura tinctoria, esse comportamento se dá em função da relação do volume e da massa do material que em situações de umidade elevada interfere nos resultados de massa específica, pois, acima de um teor de umidade de $30 \%$, considerado o ponto de saturação das fibras, a madeira não apresenta mais alterações dimensionais, apenas de peso, e essas variáveis são determinantes no resultado final da massa específica.

A massa específica básica ponderada obtida foi igual a $0,725 \mathrm{~g} / \mathrm{cm}^{3}$ sendo a madeira classificada como média de acordo com as indicações de Silveira et al. (2013). Da mesma forma, resultados semelhantes foram relatados por Alves et al. (2017) com valor de $0,703 \mathrm{~g} / \mathrm{cm}^{3}$, para Eucalyptus cloeziana, também pertencente à família Myrtacea, sendo essa comparação necessária em decorrência da ausência de informações na literatura sobre a massa específica básica especificamente do Guabiju. Ainda sobre a caracterização física do material, o resultado encontrado para a média ponderada da massa específica aparente saturada ao teor de umidade médio de $72,7 \%$ foi igual a $1,254 \mathrm{~g} / \mathrm{cm}^{3}$ e o cálculo da massa seca e massa úmida para a espécie apuraram resultados de 444 e $768 \mathrm{~kg}$, respectivamente. Valor esse 
de massa específica aparente saturada semelhante ao obtido por Candaten et al., (2018) para a madeira de Maclura tinctoria que foi de $1,134 \mathrm{~g} / \mathrm{cm}^{3}$.

Os coeficientes e as estatísticas da seleção das equações para descrever a massa específica básica e a massa específica aparente saturada de Myrcianthes pungens em função da posição relativa no sentido axial e medula-casca foram estudados e encontram-se na Tabela 2. A adição de mais variáveis independentes aos modelos não foi possível para um nível de $5 \%$ de probabilidade de erro.

TABELA 2 - Modelos de regressão obtidos pelo procedimento Stepwise para descrever massa específica básica e massa específica aparente saturada de Myrcianthes pungens em função da posição relativa no sentido axial e medula-casca (radial).

\begin{tabular}{|c|c|c|c|c|c|c|c|}
\hline \multirow{2}{*}{ Posição } & \multirow{2}{*}{ Modelo } & \multicolumn{4}{|c|}{ Estatísticas } & \multirow{2}{*}{$R_{\text {aj. }}^{2}$} & \multirow{2}{*}{$\mathrm{F}$} \\
\hline & & $b_{j}$ & Valor & $F^{\prime}$ & Prob $>F$ & & \\
\hline \multirow{3}{*}{ Axial } & \multirow{3}{*}{$\mathrm{MEb}=\mathrm{b}_{0}+\mathrm{b}_{1}\left(\mathrm{P}^{2}\right)+\mathrm{b}_{2}(\mathrm{P})$} & $\mathrm{b}_{0}$ & 0,720 & 2611,6 & $<0,001^{* *}$ & \multirow{3}{*}{0,28} & \multirow{3}{*}{11,6} \\
\hline & & $b_{1}$ & $-0,0000296$ & 11,9 & $0,0011^{* *}$ & & \\
\hline & & $b_{2}$ & 0,00201 & 5,76 & $0,0201^{*}$ & & \\
\hline \multirow{3}{*}{ Axial } & \multirow{3}{*}{$\begin{array}{c}\text { MEas }=b_{0}+b_{1}\left(P^{2}\right)+ \\
b_{2}(P)\end{array}$} & $b_{0}$ & 1,246 & 36913,1 & $<0,001^{* *}$ & \multirow{3}{*}{0,23} & \multirow{3}{*}{9,0} \\
\hline & & $b_{1}$ & $-0,0000125$ & 9,77 & $0,0029^{\star \star}$ & & \\
\hline & & $b_{2}$ & 0,00088 & 4,99 & $0,0299^{*}$ & & \\
\hline \multirow{2}{*}{ Radial } & \multirow{2}{*}{$\mathrm{MEb}=\mathrm{b}_{0}+\mathrm{b}_{1}\left(\mathrm{P}^{2}\right)$} & $b_{0}$ & 0,763 & 3955,1 & $<0,001^{\star \star}$ & \multirow{2}{*}{0,27} & \multirow{2}{*}{20,9} \\
\hline & & $b_{1}$ & $-0,0000109$ & 20,93 & $<0,001^{* *}$ & & \\
\hline \multirow{2}{*}{ Radial } & \multirow{2}{*}{ MEas $=b_{0}+b_{1}(P)$} & $b_{0}$ & 1,275 & 26248,8 & $<0,001^{* *}$ & \multirow{2}{*}{0,22} & \multirow{2}{*}{16,3} \\
\hline & & $b_{1}$ & $-0,000516$ & 16,26 & $<0,001^{* *}$ & & \\
\hline
\end{tabular}

Onde: $\mathrm{MEb}=$ massa específica básica, $\mathrm{g} / \mathrm{cm}^{3} ;$ MEas = massa específica aparente saturada, $\mathrm{g} / \mathrm{cm}^{3} ; \mathrm{P}$ = posição relativa no sentido axial e medula-casca (radial), \%; $b$ "i" = parâmetros da equação; $F^{\prime}$ = valor de $\mathrm{F}$ calculado para teste de hipótese dos parâmetros; Prob. $>\mathrm{F}=$ nível de probabilidade de erro; $\mathrm{R}_{\text {aj. }}^{2}$ = coeficiente de determinação ajustado; $\mathrm{F}=$ valor de $\mathrm{F}$ calculado para o modelo; ${ }^{* *}=$ significativo ao nível de $1 \%$ de probabilidade de erro; * = significativo ao nível de $5 \%$ de probabilidade de erro.

A partir dos resultados apresentados na Tabela 2 foi possível observar que a massa específica aparente saturada ao teor de umidade médio de $72,7 \%$ e massa específica básica foram influenciadas pelas posições radial e axial do lenho, ambas apresentando uma relação crescente da base com relação ao topo e da medula para a casca. Esse mesmo comportamento ocorreu no estudo de Silva et al. (2015) para $58 \%$ das espécies tropicais estudadas, onde os autores explicaram que isso se deu em função de fatores edáficos, topográficos, clima, entre outros, além das características internas da própria árvore e espécie. Cabe ressaltar que o teor de umidade não apresentou diferenças estatísticas nos sentidos base-topo e medulacasca, ou seja, não foram selecionadas variáveis para descrever essa propriedade ao nível de $5 \%$ de probabilidade de erro.

Ao observar esses resultados na Figura 1 verifica-se que a massa específica básica evidenciou uma tendência de redução ao longo do fuste (Figura 1A), corroborando com os resultados observados por Silva et al. (2015), para a madeira 
de Aspidosperma subincanum, e por Gallio et al. (2016) estudando a espécie de Eucalyptus benthamii. Esse comportamento provavelmente ocorre pela porção do lenho juvenil ser maior próxima ao topo, o que confere a madeira menor resistência, característica essa que está relacionada com a massa específica da madeira.

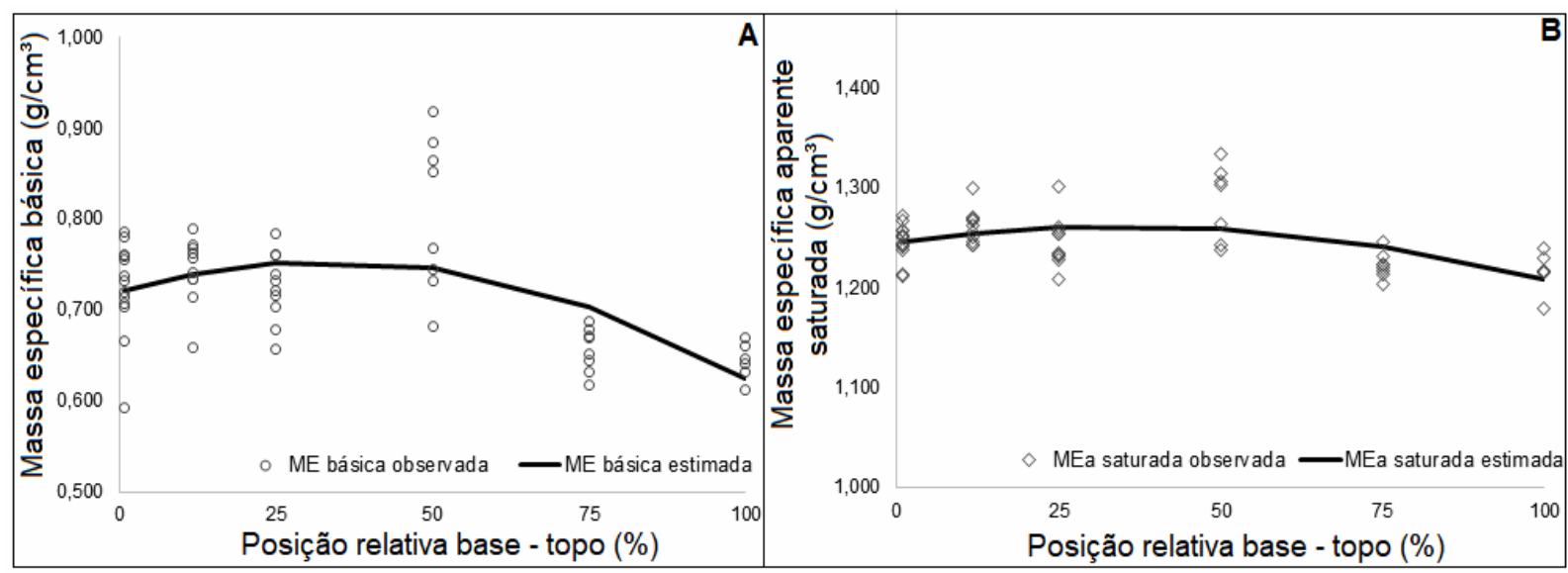

FIGURA 1 - Variação axial da massa específica básica (A) e da massa específica aparente saturada ao teor de umidade médio de $72,7 \%$ (B).

Já a massa específica aparente saturada ao teor de umidade médio de 72,7 $\%$ no sentido axial (Figura 1B) apresentou uma tendência mais homogênea de distribuição, porém, pode-se notar que houve uma redução dessa propriedade próxima ao topo. Essa variação decrescente também foi observada por Candaten et al. (2018) para a madeira Maclura tinctoria, e as menores propriedades físicas de massa específica aparente saturada obtidas ocorreram na porção final do tronco.

Com relação aos resultados apurados para a massa específica básica no sentido radial (Figura 2A), esses se mostraram maiores próximos a medula e reduziram no sentido da casca. Em função da literatura inexistente a respeito dessa espécie, ao fazer outras comparativas o comportamento difere, sendo que nos estudos de Bonduelle et al. (2015) com a madeira de Teca, por exemplo, os valores foram superiores próximos a casca. Esses autores explicaram que as fibras, os traqueoides e a parede celular implicam na massa específica em função da largura e/ou tamanho dos mesmos alterar no volume do material quando submetido a condições de umidade.

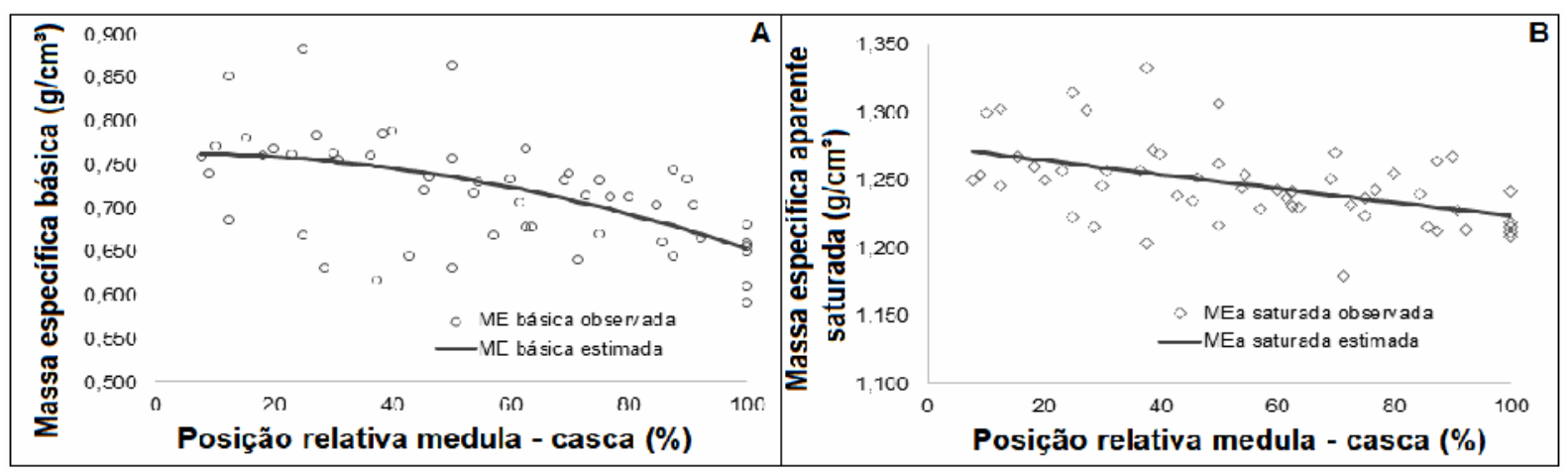

FIGURA 2 - Variação radial da massa específica básica (A) e da massa específica aparente saturada ao teor de umidade médio de $72,7 \%$ (B). 
No caso do presente estudo, por se tratar de uma árvore adulta, outra variável que pode ter influenciado no resultado da massa específica na região da medula, foi a presença de cerne, região que possui maior quantidade de extrativos e lignina (HILLIG et al., 2018), conferindo a madeira uma maior resistência mecânica e, consequentemente, uma maior massa específica. Além disso, cada espécie possui suas especificidades quanto as características químicas e físicas, o que resulta em diferentes comportamentos para um mesmo parâmetro (FONTE et al., 2017).

$A$ variação da massa específica aparente saturada no sentido medula-casca (Figura 2B) foi linearmente decrescente a partir da equação estimada, resultante da estagnação do volume do material na condição saturada e aumento do peso, conferindo maiores valores dessa propriedade próximos a medula, indicando teores de umidade elevados na região próxima à casca.

\section{CONCLUSÕES}

O teor de umidade não apresenta alterações nas posições medula-casca e base-topo, nessas mesmas condições, as propriedades físicas de massa específica básica e massa específica aparente saturada ao teor de umidade médio de 72,7 \% são influenciadas.

A massa específica básica e aparente saturada apresentam valores maiores próximos a base e menores na porção final do fuste. No sentido medula-casca essas propriedades tendem a reduzir em direção à casca na madeira de Myrcianthes pungens. A madeira dessa espécie é classificada como de média densidade.

\section{REFERÊNCIAS}

ABNT - Associação Brasileira de Normas Técnicas. NBR 7190/97: Estruturas de madeira. Rio de Janeiro, p. 107, 1997.

ALVES, R. C.; OLIVEIRA, A. L. C.; CARRASCO, E. V. M. Propriedades Físicas da Madeira de Eucalyptus cloeziana F. Muell. Floresta e Ambiente, Seropédica, v. 24, 2017. Disponível em: < http://www.scielo.br/pdf/floram/v24/2179-8087-floram-21798087015312.pdf>. doi: 10.1590/2179-8087.015312.

AMERICAN SOCIETY FOR TESTING AND MATERIALS. ASTM D 143-94: Standard test methods for small clear specimens of timber. Philadelphia, 1995.

BONDUELLE, G. M.; IWAKIRI, S.; TRIANOSKI, R.; PRATA, J. G.; ROCHA, V. Y. da. Análise da massa específica e da retratibilidade da madeira de Tectona grandis nos sentidos axial e radial do tronco. Revista Floresta, Curitiba, v. 45, n. 4, p. 971-680, 2015. Disponível em: <https://revistas.ufpr.br/floresta/article/view/31991/26918>. doi:10.5380/rf.v45i4.3191.

CANDATEN, L.; MANGINI, T.; BANDERA, E.; TREVISAN, R.; COSTA, H. W. D. Relação da massa específica e teor de umidade da madeira de Maclura tinctoria ao longo do fuste. Enciclopédia Biosfera, Goiânia, v. 15, n. 28, p. 845-854, 2018. Disponível em: <http://www.conhecer.org.br/enciclop/2018B/agrar.htm>. doi: 10.18677/EnciBio_2018B68.

DIAS, D. da C.; COLODETTE, J. L.; THIERSCH, C. R.; LEITE, H. G.; GOMIDE, J. L. Uso da técnica de resistografia e de variáveis dendrométricas na modelagem da densidade básica de povoamentos clonais de Eucalyptus. Ciência Florestal, Santa 
Maria, v. 27, n. 2, p. 609-619, 2017. Disponível em: <http://www.scielo.br/scielo.php?pid=S198050982017000200609\&script=sci_abstrac t\&tlng=en>. doi: 10.5902/1980509827746.

FONTE, A. P. N.; TRIANOSKI, R.; IWAKIRI, S.; ANJOS, R. A. M. dos; Propriedades físicas e químicas da madeira de cerne e alburno de Cryptomeria japonica. Revista de Ciências Agroveterinárias, Lages, v. 16, n. 3, p. 277-285, 2017. Disponível em: <http://www.revistas.udesc.br/index.php/agroveterinaria/article/view/2238117116320 17277/pdf>. doi: $10.5965 / 223811711632017277$.

FRIEDERICHS, G.; BRAND, M. A.; CARVALHO, A. F. de.; KÜSTER, L. C. Qualidade da madeira e do carvão vegetal de bracatinga (Mimosa scabrella Benth.). Ciência da Madeira, Pelotas, v. 6, n. 2, p. 79-87, 2015. Disponível em: <https://periodicos.ufpel.edu.br/ojs2/index.php/cienciadamadeira/article/view/4132/42 67>. doi: 10.12953/2177-6830/rcm.v6n2p79-8.

GALLIO, E.; SANTINI, É. J.; GATTO, D. A.; SOUZA, J. T. de; RAVASI, J. et al. Caracterização tecnológica da madeira de Eucalyptus benthamii Maiden et Cambage. Scientia Agraria Paranaensis, Marechal Cândido Rondon, v. 15, n. 3, p. 244-250, 2016. doi: 10.18188/1983-1471/sap.v15n3p244-250.

HILLIG, É.; DIGNER, T.; DIAS, A. N. Propriedades físico-mecânicas da madeira de uva-do-japão. Pesquisa Florestal Brasileira, Colombo, v. 38, p. 1-6, 2018. Disponível em: <https://pfb.cnpf.embrapa.br/pfb/index.php/pfb/article/view/1195>. doi: 10.4336/2018.pfb.38e201601195.

JUIZO, C. G. F.; LOIOLA, P. L.; ZEN, L. R.; MARCHESAN, R.; CARVALHO, D. E. et al. Variação radial das propriedades físicas da madeira de Pinus patula plantados em Moçambique. Pesquisa Florestal Brasileira, Colombo, v. 35, n. 83, p. 285-292, 2015.

em:

<https://pfb.cnpf.embrapa.br/pfb/index.php/pfb/article/view/771>. doi:

10.4336/2015.pfb.35.83.771.

MATTOS, B. D.; GATTO, D. A.; STANGERLIN, D. M.; CALEGARI, L.; MELO, R. R.; SANTINI, E. J. Variação axial da densidade básica da madeira de três espécies de gimnospermas Revista Brasileira de Ciências Agrárias, Pernambuco, v. 6, n. 1, p. 121-126, $2011 . \quad$ Disponível em: <http://www.redalyc.org/pdf/1190/119018527018.pdf>. doi: 10.5039/agraria.v6i1a1080.

OSBORNE, N. L. Estimating the density of coast Douglas-fir wood samples at diferente moisture contents using medical X-ray computed tomography. Computers and Electronics in Agriculture, v. 127, p. 50-55, 2016. doi: 10.1016/j.compag.2016.06.0030168-1699.

SILVA, C. J. da; VALE, A. T. do; MIGUEL, E. P. Densidade básica da madeira de espécies arbóreas de Cerradão no estado de Tocantins. Pesquisa Florestal Brasileira, Colombo, p. 64-75, 2015. Disponível em: < http://pfb.cnpf.embrapa.br/pfb>. doi: 10.4336/2015.pfb.35.82.822. 
SILVEIRA, L. H. C.; REZENDE, A. V.; VALE, A. T. Teor de umidade e densidade básica da madeira de nove espécies comerciais amazônicas. Acta Amazônica, Manaus, v. 43, n. 2, p.179 - 184, 2013. doi: 10.1590/S0044-59672013000200007

TRAUTENMÜLLER, J. W.; BALBINOT, R.; BORELLA, J.; TREVISAN, R.; BALESTRIN, D. et al. Variação longitudinal da massa específica básica da madeira de Cordia americana e Alchornea triplinervia. Ciência Rural, Santa Maria, v. 44, n. 5, p. 817-821, 2014. Disponível em:<http://www.redalyc.org/html/331/33130634009/>. doi: 10.1590/S010384782014000500009.

VITAL, B.R. Métodos de determinação da densidade da madeira. Viçosa, p. 24, 1984. (Boletim Técnico, 2).

WILSON P.G.; O'BRIEN, M.M.; GADEK, P.A.; QUINN, C.J. Myrtaceae revisited: a reassessment of intrafamilial groups. American Journal of Botany, Columbus, n.88, p. 2013-2025, 2001. Disponível em: < https://bsapubs.onlinelibrary.wiley.com/doi/pdf/10.2307/3558428>. doi: $10.2307 / 3558428$.

ZAQUE, L. A. M.; FERREIRA, M. D.; MELO, R. R. Variação radial e longitudinal da massa específica básica da madeira de Araucaria angustifolia com diferentes idades. Pesquisa Florestal Brasileira, Colombo, v. 38, p. 1-5, 2018. Disponível em: <https://pfb.cnpf.embrapa.br/pfb/index.php/pfb/article/view/e01368/663>. doi: 10.4336/2018.pfb.38e201601368. 